\title{
Effect of Mixing Strategy on the Structure-Properties of the PLA/PBAT Blends Incorporated with $\mathrm{CNC}$
}

\author{
Deniz Sema Sarul ${ }^{1}$, Dogan Arslan ${ }^{2}$, Emre Vatansever ${ }^{1}$, Yusuf Kahraman ${ }^{2}$, Ali Durmus ${ }^{3}$, Reza Salehiyan ${ }^{4}$ \\ and Mohammadreza Nofar ${ }^{1,2, *}$ \\ ${ }^{1}$ Polymer Science and Technology Program, Institute of Science and Technology, Istanbul Technical University, Istanbul, 34469, \\ Turkey \\ ${ }^{2}$ Metallurgical \& Materials Engineering Department, Faculty of Chemical and Metallurgical Engineering, Istanbul Technical \\ University, Istanbul, 34469, Turkey \\ ${ }^{3}$ Department of Chemical Engineering, Faculty of Engineering, Istanbul University-Cerrahpasa, Istanbul, 34320, Turkey \\ ${ }^{4}$ Department of Materials Research and Technology, Luxembourg Institute of Science and Technology, Esch-sur-Alzette, 4362, Luxembourg \\ ${ }^{*}$ Corresponding Author: Mohammadreza Nofar. Email: nofar@itu.edu.tr
}

Received: 10 April 2021 Accepted: 02 June 2021

\begin{abstract}
Polylactide (PLA)/poly (butylene adipate-co-terephthalate) (PBAT) blend nanocomposites including $3 \mathrm{wt} \%$ of cellulose nanocrystals (CNCs) were prepared by melt compounding method in a twin-screw extruder and an internal mixer. Blend nanocomposites were formulated by diluting three different masterbatches prepared by solution casting method that contained $7 \mathrm{wt} \%$ of CNC. These masterbatches were: $(\mathrm{m} 1)$ PLA/PBAT/CNC masterbatch; (m2) PLA/CNC masterbatch; and (m3) PBAT/CNC masterbatch. These were to explore how different preparation methods affect the dispersion and localization of CNC and hence the properties of PLA/PBAT/CNC blend nanocomposites. Scanning electron microscopy (SEM) was used to study the structural changes of the blends. Rheological properties of PLA/PBAT blends and PLA/PBAT/CNC blend nanocomposites were also investigated. In the samples prepared by internal mixer, the rheological behavior of blend nanocomposite prepared through premixing of CNC particles with PLA showed a transition from liquid-like to a gel-like behavior. According to the rheological results and differential scanning calorimetry (DSC) analysis, it was found that the CNC overall enhanced the viscoelastic properties of blends and improved the PLA crystallization, respectively. Dynamic mechanical analysis (DMA) illustrated that the incorporation of CNC also enhanced the elastic modulus of PLA/PBAT blends specifically above the glass transition temperature of PLA. The expected improvements in mechanical properties did not occur due to the possible existence of residual solvent in the blends.
\end{abstract}

\section{KEYWORDS}

Polylactide; poly(butylene adipate-co-terephthalate); cellulose nanocrystals blend; nanocomposite; morphology; rheology

\section{Introduction}

Bio-based and/or biodegradable polymer studies have remarkably increased in recent years due to the concerns about environmental effects of petroleum-based polymers and consumption of crude oil 
reserves, promptly. Polylactide (PLA) is one of the most promising bio-based polymers due to its $100 \%$ biobased synthetic route and fully biodegradable nature, reasonable mechanical strength, and commercial availability [1-4]. However, PLA lacks some crucial characteristics like flexibility, melt strength, and fast crystallization rate $[3,5]$. The economically and technically most viable method to overcome these drawbacks is the blending of PLA with high impact bioplastics like poly (butylene adipate-coterephthalate) (PBAT). Some studies have shown that blending of PLA with PBAT can improve the toughness of PLA, but significantly decreases the modulus and mechanical strength of PLA. It has also been reported that PLA/PBAT blends are generally completely immiscible $[3,6,7]$.

The addition of solid nanoparticles into immiscible polymer blends is frequently employed for imparting a somewhat compatibility between two polymers and achieves balance between toughness and stiffness. However, it has been well-documented that the improvements in blend properties are highly dependent on the localization of nanofillers into blend. Nanofillers can localize into one polymer or at the interface between the two polymers [8-11]. If the nanofillers are localized in the dispersed phase, size of dispersed phase droplets increases due to the viscosity elasticity enhancement of the dispersed phase [12,13]. On the other hand, if nanoparticles are localized in the matrix phase, size of dispersed phase decreases and the modulus and strength of blend can increase as well as impact properties [10,14]. Eventually, it has been reported that the distribution of nanofillers at the interface decreases the dispersed phase size and increases the interfacial interactions between phases $[9,15,16]$.

The selective localization of nanofillers strongly depends on thermodynamic and kinetic parameters. The thermodynamic factors refer to the surface energies of the nanofiller and the polymer components. According to the thermodynamic effect, nanofillers tend to be localized in the phase which they have the lowest interfacial tension with in order to mitigate the interfacial energy between the polymers. Kinetic parameters are mainly related to physical issues of mixing process, such as mixing strategy, melt viscosity of polymers, melt compounding time, shear rate and shape of nanoparticles [14,17-20]. Several studies have investigated the role of kinetic and thermodynamic parameters on the localization of nanofillers in PLA/PBAT blends. Nofar et al. [11] found that in the PLA/PBAT/clay nanocomposite prepared in an internal mixer, the nanoclay $(1 \mathrm{wt} \%)$ is localized at the interface between polymers as predicted from thermodynamics. They also noted that with the increasing clay content, the excess amount of clay could migrate to the PLA matrix. Dil et al. [9] investigated the distribution of nano-silica in the PLA/PBAT blend using two different mixing strategies. Although different mixing strategies were applied, it was assumed that nano-silica preferred to migrate to the PBAT phase as predicted from thermodynamics. However, silica nanoparticles could be found in the PLA or at the interface thanks to the dominant effect of thermodynamics $v s$. kinetics. When the nano-silica content increased to $3 \mathrm{wt} \%$, the dispersed phase morphology of PLA/PBAT (70/30) blend turned into a co-continuous structure. They reported that the morphological inversion led to increase in the elongation at break and impact strength values of blends. In another study, it was shown that carbon nanotubes (CNTs) were always located within PBAT phase in the PLA/PBAT blends with different blend compositions [21]. It was also determined that high polarity nanowire particles can only migrate to polar phase or interfaces in the case of long mixing time or high shear rate [22]. It was also reported that graphene sheets were dispersed at PBAT phase, while the nanoclay sheets were dispersed at the interface and PLA matrix [23].

In this study, cellulose nanocrystal (CNC) nanoparticles are introduced into PLA/PBAT blends due to its superior properties and its abundancy in nature, high modulus, high strength, renewability, biocompatibility, nontoxicity, large surface area and low density. Thanks to these properties, CNC is an alternative to inorganic reinforcements as it could improve the properties of polymers at low content [24-28]. CNC is hydrophilic and one-dimensional (1D) fibrous material. This could bring about CNC agglomerations in a polymer matrix when simultaneous melt mixing methods are used [29-32]. Therefore, the solvent dissolution is shown to be one of the most efficient methods to disperse CNC in polymers such as PLA and 
PBAT [32-36]. It has been reported that a combination of solution casting and melt mixing methods may result in a good level of CNC dispersion in PLA and PBAT [32,35,37]. Mohammadi et al. [37] explored the localization of CNCs in PLA (amorphous and semicrystalline)/PBAT blend nanocomposites. Corresponding nanocomposites were prepared through firstly solution casting with different mixing strategies, and then melt mixing using an internal mixer. Regardless of the initial localization of the CNCs, they were found to be inside the PBAT droplets. This in turn, led to PBAT droplet size reduction and finer morphology in the blend nanocomposites. Pracella et al. [38] prepared PLA/poly (vinyl acetate) (PVAc)/CNC blend nanocomposites by mixing PVAc/CNC masterbatches with various amounts of PLA. They observed that the PLA/PVAc/CNCs blend nanocomposites showed better CNC distribution and thus enhanced tensile properties and higher thermal resistance compared to the PLA/CNC nanocomposites. This increase in the mechanical properties was attributed to improved interfacial interactions between polymer matrix and filler surfaces. Shakouri et al. [39] investigated morphological and physical properties of PLA/thermoplastic polyurethane (TPU) (80/20) blends containing geometrically different cellulose nanocrystals (CNCs) prepared with melt mixing method. They found that both spherical and cylindrical CNCs were finely dispersed in the PLA matrix and/or interface, while poorly dispersed rod-like CNCs were mostly found in the TPU phase. The blend of PLA/TPU with spherical CNCs showed higher toughness than the neat blend. A similar effect was observed for the cylindrical CNCs. On the other hand, they also reported that the rod-like CNCs decreased the blend toughness. Bitinis et al. [40] showed that the viscosity and storage modulus values of PLA/natural rubber (NR) blends were increased with $\mathrm{CNC}$ incorporations when both solution casting and melt mixing methods were used consecutively. This is while, the direct extrusion method did not yield an increase in these parameters. However, combining solvent casting and extrusion could not improve the mechanical properties that decreased by blending PLA with NR. Heshmati et al. [41-42] found that the CNC tends to be situated in the polyamide (PA) polymer in the PLA/PA11 (50/50) blend. When the polyethylene oxide (PEO)-coated CNC was introduced into the PLA/PA11 blend, a co-continuous morphology was obtained with CNCs in the PLA phase.

Comparison of PLA/PBAT/CNC blends prepared by different process method has not been studied in the literature so far. Thereby, the main objective of this article is how different preparation methods affect the dispersion and localization of $\mathrm{CNC}$ and hence the properties of PLA/PBAT/CNC blend nanocomposites. In our previous study [43], it was observed that, in PLA/PBAT blends prepared with solution casting (SC) and melt processing combination, CNCs preferred to be localized in PBAT minor phase. This was while with increasing CNC content, the excessive CNCs tended to migrate from PBAT to the interface and PLA matrix. In addition, it was also reported that the expected mechanical properties were not observed possibly due to the involvement of residual solvents although superior rheological results were obtained with the increasing amount of CNC. In this study, we aimed to explore the morphological, rheological, crystallization, thermomechanical, and mechanical properties of PLA/PBAT blends and blend nanocomposites containing $3 \mathrm{wt} \% \mathrm{CNC}$ prepared through dilution of solution casted masterbatch via melt mixing method using (a) a twin-screw extruder (TSE) and (b) an internal mixer (IM). The employed melt processing strategies are referred to as (a) mTSE and (b) mIM, respectively. In this context, three different masterbatches, i.e., (m1) PLA/PBAT/CNC masterbatch; (m2) PLA/CNC masterbatch; and (m3) PBAT/CNC masterbatch, were incorporated for dilution processes through the two noted melt mixing processes.

\section{Experimental}

\subsection{Materials}

A commercial grade of PLA (Ingeo 3001D) was kindly supplied from NatureWorks LLC. The PBAT (Ecoflex ${ }^{\circledR}$ F Blend C1200) was provided from BASF, a grade for blown or cast film processing, with the 
weight average molecular weight $\left(\mathrm{M}_{\mathrm{w}}\right)$ and polydispersity index of $126 \mathrm{~kg} / \mathrm{mol}$ and 1.75 , respectively. Spraydried CNCs prepared with sulfuric acid hydrolysis of wood pulp were provided by CelluForce (Montreal, Canada). It has been reported that these CNCs possess average length, width, and aspect ratio of around $165 \mathrm{~nm}, 13 \mathrm{~nm}$, and 12.7, respectively [44]. A reagent grade dimethylformamide (DMF) with a purity of 99\% was purchased from Sigma-Aldrich.

\subsection{Blend Nanocomposites Preparation}

\subsubsection{Solution Casting Method}

The PLA/PBAT/CNC blend nanocomposites were made through dilution of the solution casted masterbatches containing $7 \mathrm{wt} \%$ of $\mathrm{CNC}$ using a twin-screw extruder, or an internal mixer that were proposed by Bagheriasl et al. [32]. Three different masterbatches used for dilution were: $(\mathrm{m} 1)$ PLA/PBAT/CNC masterbatch, (m2) PLA/CNC masterbatch, and (m3) PBAT/CNC masterbatch. Therefore, to improve the CNC dispersion, masterbatches with the CNC content of $7 \mathrm{wt} \%$ were first prepared by solvent casting [32]. The CNCs were dispersed into $100 \mathrm{~mL}$ of DMF on a magnetic stirrer and the sonicated for $2 \mathrm{~h}$ in a water bath sonicator. The PLA and PBAT were then added to the $\mathrm{CNC} / \mathrm{DMF}$ suspension according to the type of masterbatch. The mixture was rigorously stirred at $70^{\circ} \mathrm{C}$ on magnetic stirrer for 150 min until full dissolution of polymer pellets. The solution was finally casted into petri glass dishes, dried at $85^{\circ} \mathrm{C}$ for $36 \mathrm{~h}$ under vacuum, and grinded. Ground samples were then dried at $85^{\circ} \mathrm{C}$ for $48 \mathrm{~h}$ under vacuum for excess solvent removal. The current described method firstly introduced by Carreau group [34] yielded finely dispersed CNCs in PLA matrix.

\subsubsection{Melt Mixing Method}

The above noted three masterbatches were diluted through two different melt mixing methods to obtain blend nanocomposites with the composition of $3 \mathrm{wt} \% \mathrm{CNC}$ and $25 \mathrm{wt} \%$ PBAT concentration. In the first method, a corotating twin-screw extruder (TSE) (Microlab TSE-Rondol) with L/D of 20 was used. The extruder was operated with a screw-speed of $50 \mathrm{rpm}$ and temperature profile of 120,150,170,170, and $170^{\circ} \mathrm{C}$ from the feeding zone to die. In the other method, an internal mixer (IM) (RTX-M40 Melt Mixer) was used at $50 \mathrm{rpm}$ for $10 \mathrm{~min}$ at a temperature of $170^{\circ} \mathrm{C}$. The melt processed samples were dried in a vacuum oven for overnight at $50^{\circ} \mathrm{C}$. The samples were then compression molded at $180^{\circ} \mathrm{C}$ to prepare various test specimens for characterization studies. Tab. 1 presents the samples names according to the preparation method and composition.

Table 1: The composition details of all samples. Samples 1 and 2 are adopted from our previous study [43]

\begin{tabular}{llllll}
\hline \multicolumn{2}{l}{ Samples } & $\begin{array}{l}\text { PLA/PBAT } \\
\text { weight ratio }\end{array}$ & CNC content (wt\%) & $\begin{array}{l}\text { Masterbatch } \\
\text { strategy }\end{array}$ & Method \\
\hline 1 & PLA/PBAT & $75 / 25$ & 0 & - & Direct TSE \\
2 & PLA/PBAT/CNC & $72 / 25$ & 3 & $\mathrm{~m} 1$ & SC+TSE \\
3 & (PLA/CNC)/PBAT & $72 / 25$ & 3 & $\mathrm{~m} 2$ & $\mathrm{SC}+\mathrm{TSE}$ \\
4 & (PBAT/CNC)/PLA & $72 / 25$ & 3 & $\mathrm{~m} 3$ & $\mathrm{SC}+\mathrm{TSE}$ \\
5 & $\mathrm{PLA} / \mathrm{PBAT}$ & $75 / 25$ & 0 & - & Direct IM \\
6 & $\mathrm{PLA} / \mathrm{PBAT} / \mathrm{CNC}$ & $72 / 25$ & 3 & $\mathrm{~m} 1$ & $\mathrm{SC}+\mathrm{IM}$ \\
7 & (PLA/CNC)/PBAT & $72 / 25$ & 3 & $\mathrm{~m} 2$ & $\mathrm{SC}+\mathrm{IM}$ \\
8 & (PBAT/CNC)/PLA & $72 / 25$ & 3 & $\mathrm{~m} 3$ & $\mathrm{SC}+\mathrm{IM}$ \\
\hline
\end{tabular}




\subsection{Morphological Analysis}

The molded samples were cryofractured in liquid nitrogen and coated with platinum before SEM analysis. A field emission gun scanning electron microscope (SEM JEOL 7600F FEG, Japan) was used to determine the morphological behavior of the blends and blend nanocomposites.

\subsection{Rheological Analysis}

The rheological properties of the blends and blend nanocomposites were measured at $170^{\circ} \mathrm{C}$ using a Modular compact rheometer (MCR-301, Anton Paar-Austria) with a $25 \mathrm{~mm}$ parallel-plate geometry and $1 \mathrm{~mm}$ gap. The linear viscoelastic behavior of the blend nanocomposites was measured from small amplitude oscillatory shear (SAOS) tests. SAOS tests were conducted from high to low frequencies at the strain amplitude of 0.05 , which was within the linear viscoelastic region. Stress-growth experiments were performed under rotational shear flow at $0.01 \mathrm{~s}^{-1}$. In order to avoid degradation, all experiments were carried out under nitrogen gas.

\subsection{Differential Scanning Calorimetry (DSC) Analysis}

The melting and crystallization behaviors and the transition temperatures of blends and blend nanocomposites were explored using a DSC, Q2000, TA Instruments under nitrogen atmosphere. The samples were exposed to heat $/ \mathrm{cool} /$ heat cycles between $30^{\circ} \mathrm{C}$ and $200^{\circ} \mathrm{C}$. While the heating cycles were conducted at a heating rate of $10^{\circ} \mathrm{C} / \mathrm{min}$, the cooling was performed at a rate of $2^{\circ} \mathrm{C} / \mathrm{min}$. The degree of crystallinity, $X_{\mathbf{c}}$, was calculated using the following Eqs. (1) and (2):

$X_{c}^{\text {heating }}=\frac{\left(\Delta H_{m}-\Delta H_{c c}\right) \times 100}{w_{P L A} \Delta H_{m}^{0}}$

$X_{c}^{\text {cooling }}=\frac{\left(\Delta H_{c}\right) \times 100}{w_{P L A} \Delta H_{m}^{0}}$

where $\Delta \mathrm{H}_{\mathrm{m}}, \Delta \mathrm{H}_{\mathrm{c}}, \Delta \mathrm{H}_{\mathrm{cc}}$, and $\Delta \mathrm{H}_{\mathrm{m}}^{\mathrm{o}}$ are, respectively, the heat enthalpies of melting, melt crystallization, cold crystallization, and melting of $100 \%$ crystalline PLA (i.e., $93.6 \mathrm{~J} / \mathrm{g}$ ) and $w_{\mathrm{PLA}}$ is PLA weight fraction [45].

\subsection{Dynamic Mechanical Analyzer (DMA)}

The thermomechanical properties of the blends and blend nanocomposites were analyzed in a single cantilever mode from 25 to $120^{\circ} \mathrm{C}$ using a Perkin Elmer DMA 8000. The dimensions of rectangular samples were 50,10, and $2 \mathrm{~mm}$. The deformation amplitude, applied frequency, and heating rate were, respectively, $30 \mu \mathrm{m}, 1 \mathrm{~Hz}$, and $3^{\circ} \mathrm{C} / \mathrm{min}$.

\subsection{Mechanical Analysis}

Tensile properties of the blends and blend nanocomposites were analyzed using an Instron 8801 with $25 \mathrm{kN}$ load cell model drawing device. The drawing speed was $5 \mathrm{~mm} / \mathrm{min}$. The prepared samples were dog bone shaped with length, width, and thickness of 20,4 and $3 \mathrm{~mm}$, respectively. Tensile strength, Young's modulus, elongation at break and energy at break values of the samples were calculated from the stress-strain graphs. The tensile test was repeated 5 times for each sample and their average values were reported.

Izod impact strength of the blends and blend nanocomposites was measured using a Zwick Roell impact device with a $1 \mathrm{~J}$ pendulum falling at an angle of $160^{\circ}$. The prepared samples were rectangular with length, 
width, and thickness of 80,10 and $4 \mathrm{~mm}$, respectively. All samples were notched $2 \mathrm{~mm}$ at an angle of $45^{\circ}$ before testing. The impact test was repeated 6 times for each sample and their averages values were reported.

\section{Results and Discussion}

\subsection{CNC Localization in PLA/PBAT Blends in an Equilibrium State}

In the previous study [43], according to the calculations, it was determined that CNCs in PLA/PBAT blends thermodynamically prefer to be localized in PBAT phase.

\subsection{Morphological Analysis}

Fig. 1 shows the SEM images of PLA/PBAT blends and PLA/PBAT blend nanocomposites containing $3 \mathrm{wt} \%$ of CNC prepared through three different masterbatches and two different melt mixing methods (mTSE and $\mathrm{mIM}$ ). Neat PLA/PBAT (75 wt $\% / 25 \mathrm{wt} \%$ ) blends were prepared directly via TSE or IM. Generally, regardless of the type of used masterbatch, it was observed that the droplet size of dispersed phase in the neat blend prepared through IM was larger than that of the blend prepared by TSE. One can easily assume that the TSE and IM generate different shear forces as a result of device geometry and operation conditions. The IM provides a predominantly simple shear flow between the sample and the chamber walls, as well as a low elongation field, while the TSE loads a strong shear and elongation flow upon the sample [19]. Therefore, it could be deduced that the elongational flow field of TSE is particularly effective in reducing the droplet size of PBAT. It was also found that droplet size of dispersed phase in the blend nanocomposite prepared with the $\mathrm{m} 1$ strategy did not change regardless of both melt mixing methods. This can be explained by the localization of CNCs throughout the blend. On the other hand, large droplets were observed in the blend nanocomposite prepared with the mTSE and $\mathrm{m} 2$ strategy. During the melt mixing, the intensive shear mixing condition is capable of breaking up the droplets. Further, the droplets can collide again to merge into bigger droplets. It can be assumed that these large droplets may lead to re-agglomeration of CNCs in the PLA continuous matrix phase since the CNCs are already pre-dispersed in the PLA matrix. Small droplets were found in the blend nanocomposite prepared with the IM and $\mathrm{m} 2$ strategy. This could be originated from the lesser extent of CNCs re-agglomeration, since the internal mixer yields lower shear forces than the extruder. It is also worth mentioning that residence time is another distinctive parameter in defining the morphologies. IM provides longer mixing time than the TSE, therefore, in a longer mixing process the larger agglomerates have enough time to reagglomerate. In the $\mathrm{m} 3$ strategy where CNC particles are pre-mixed with PBAT, there was no enough time in mTSE for CNC to migrate from PBAT to PLA. The particular localization of CNC into PBAT phase increases the PBAT droplet size by increasing the its viscosity and elasticity. It could be inferred that the high viscosity of PBAT resists against the shear deformations and eventually breaking up the droplets during mixing. Moreover, droplets may collide from the free-CNC interfaces and afford the coalescence which leads to increase in their size. On the other hand, in the $\mathrm{m} 3$ strategy prepared with IM, it was observed that the droplets were small because there might be enough time for the CNC migration from PBAT to PLA or localization at the interface. CNC nanoparticles localized in the PLA matrix, can decrease the viscosity ratio between PLA matrix and PBAT dispersed phase which leads to facilitated breakup of PBAT droplets. Furthermore, CNCs at the interface, can suppress the coalescence of PBAT droplets. Based on these results, it can be speculated that the kinetic parameters can play important role in determining the localization of CNC. In general, size reduction was more obvious in the IM blends, indicating the important role of mixing time on the formation of blend morphology. It is worth remembering that the SEM images could only reveal the shape and size of PBAT droplets as a result of $\mathrm{CNC}$ incorporation. This is while, understanding the state of $\mathrm{CNC}$ dispersion and their localization requires further studies. 

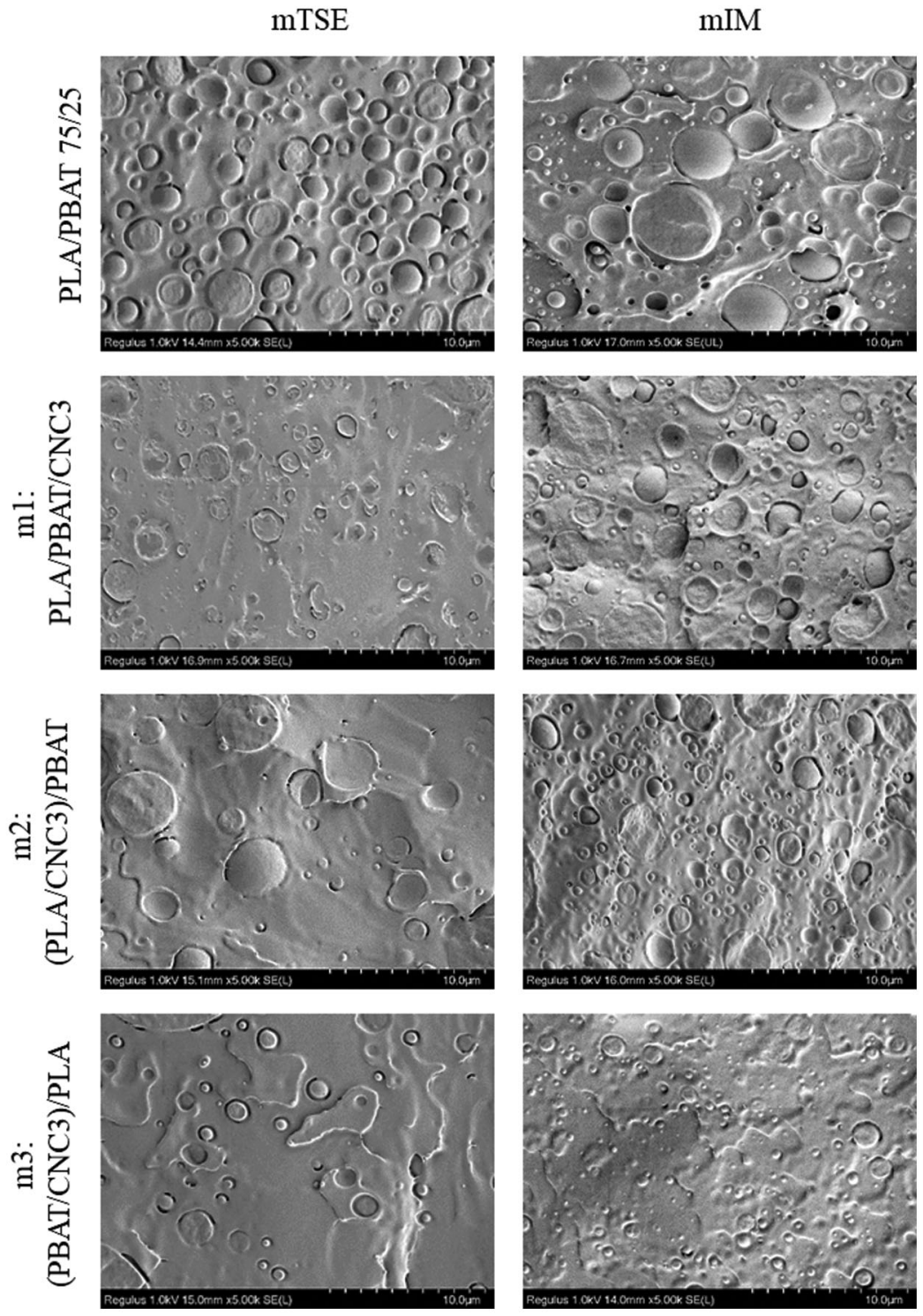

Figure 1: SEM images of samples with different mixing strategies and melt mixing methods. The image of m1: PLA/PBAT/CNC3 sample prepared through mTSE is adopted from our previous study [43]

\subsection{Rheological Analysis}

Fig. 2 compares the rheological properties of PLA/PBAT blends and PLA/PBAT blend nanocomposites containing $3 \mathrm{wt} \%$ of CNC prepared through three mixing strategies and two melt mixing method (mTSE and $\mathrm{mIM}$ ). It has been confirmed that the solution casting method preserved the good dispersion of CNC in the PLA and PBAT [33-37] and hence, blend nanocomposites containing $3 \mathrm{wt} \%$ of CNC were prepared through melt mixing of solution-casted PLA/PBAT/CNC (m1 strategy), PLA/CNC (m2 strategy) and PBAT/CNC (m3 strategy) masterbatches. 

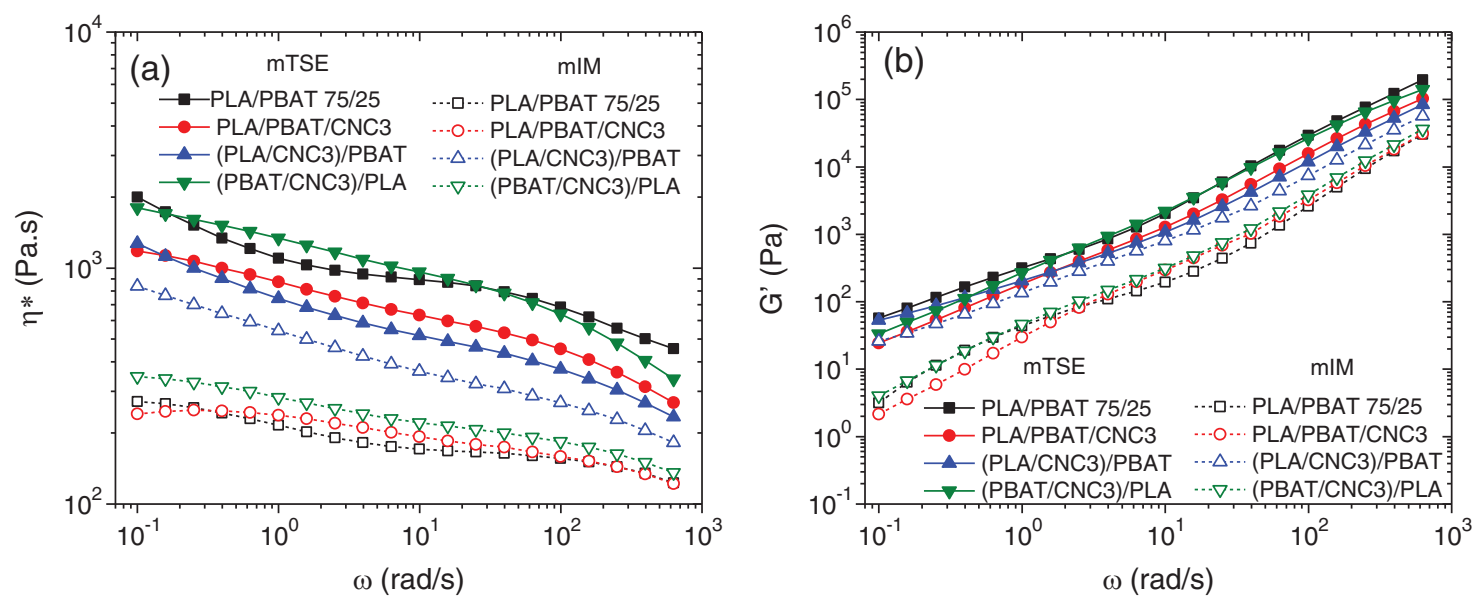

Figure 2: (a) Complex viscosity, $\eta^{*}$, and (b) storage modulus, $\mathrm{G}^{\prime}$, of the blends and blend nanocomposites with $3 \mathrm{wt} \%$ CNC. The data of PLA/PBAT and PLA/PBAT/CNC3 samples prepared through mTSE are adopted from our previous study [43]

It has been reported that shear flow significantly reduces the interaction among particles and causes significant changes in viscoelastic behavior [46]. As seen in Fig. 2, the complex viscosity, $\eta^{*}$, and storage modulus, $\mathrm{G}^{\prime}$, of the blend prepared with the IM are lower than those of the blend prepared with the TSE. In case of $3 \mathrm{wt} \%$ of $\mathrm{CNC}$ addition, it was observed that the $\eta^{*}$ and $\mathrm{G}^{\prime}$ of blend nanocomposites prepared with mTSE were higher than those of blend nanocomposites prepared with mIM. In the mTSE prepared blend nanocomposites, the viscosity $\eta^{*}$ and elastic modulus $G^{\prime}$ were lower than those of the neat PLA/PBAT blend. This could be possibly due to the presence of residual solvent in the nanocomposites while, the neat blend was prepared directly through TSE where no solvent was required.

Remembering the morphological differences between the mTSE and mIM nanocomposites, one would expect that the blends with smaller droplets exhibit higher viscoelastic values. However, there is no consistency between the viscoelastic parameters of two different blends. This can also be attributed to the different thermal histories of blends. Blends prepared via IM were exposed to longer compounding time at processing temperature which may cause lower viscosity values than those prepared in TSE. Although, it is important to note that viscoelastic improvements can be observed in the case of IM blends (slightly in $\mathrm{m} 3$ and dramatically in $\mathrm{m} 2$ ) which corresponds to the smaller droplet sizes. This can also be attributed to the formation of CNC network in PLA matrix indicated by the transition from liquid to solid-like behavior when strong interactions existed between the nanofiller and matrix. This is probably originated from the fact that CNCs are pre-mixed with PLA thus CNCs are localized in the PLA matrix. This is while, in the case of $\mathrm{m} 3$ the CNCs could partially migrate towards the PLA and interface which caused smaller droplets and hence, slightly higher viscoelastic properties. On the other hand, the viscoelastic properties reduced in the case of TSE with the CNC addition ( $\mathrm{m} 2$ and $\mathrm{m} 3$ ) specifically at low frequency region. This result indicated that TSE method failed to yield CNC network. Another reason could be related to decrease in the aspect ratio of CNC significantly as a result of intensive shearing into the TSE.

In order to examine the morphological stability of the blend and nanocomposites under constant rotational shear, stress growth experiments were also carried out under shear of $0.01 \mathrm{~s}^{-1}$. The time evolution of transient viscosity, $\eta^{+}$, of the blends and nanocomposites are shown in Fig. 3. The viscosity reduction with time could be an indication of the droplet coalescence or thermal degradation of PLA [37]. It can be seen that $\eta^{+}$enhanced with $3 \mathrm{wt} \%$ addition of $\mathrm{CNC}$, irrespective of the processing method and mixing strategy. These enormous increases can be attributed to improvement in the dispersion of 
nanoparticles under shear flow. However, there was no significant increase in $\eta^{+}$of the blend nanocomposite prepared through mTSE with $\mathrm{m} 3$ strategy. Therefore, it can be assumed that the disperse phase starts to coalesce due to high shear flow in TSE. In general, the growth in the case of IM blend nanocomposites is shown to be more pronounced. This is an indication of facilitated de-agglomeration of CNCs in a low viscosity polymer melt. The lower viscosities of neat mIM PLA/PBAT blend can clearly be seen compared to that of the neat mTSE blend. The fact that the viscosities of the neat blends do not show the growth behavior is an indication of the absence of any CNC clusters to be de-agglomerated.

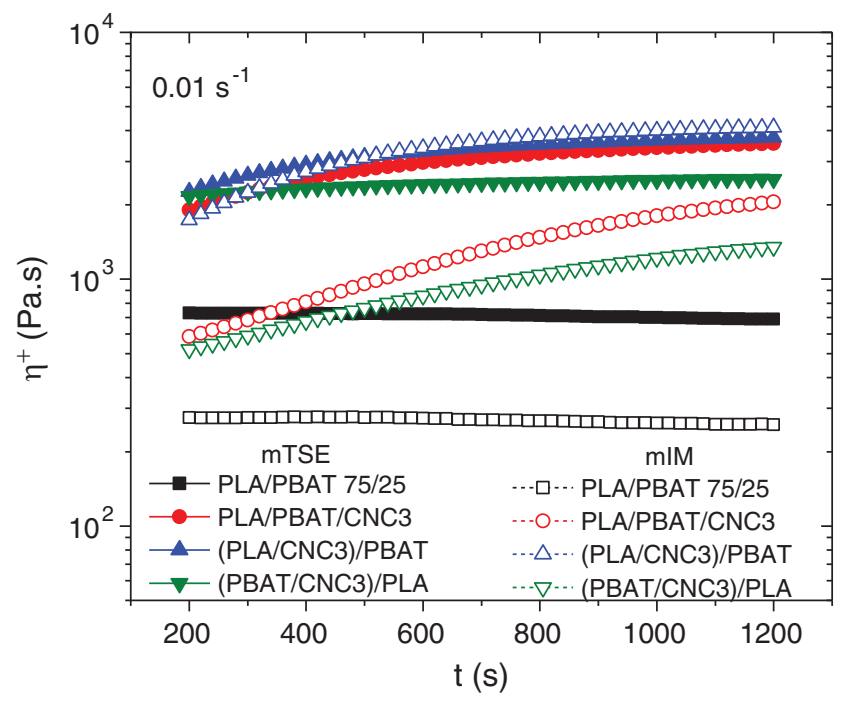

Figure 3: Stress growth results of the blends and $3 \mathrm{wt} \% \mathrm{CNC}$ loaded nanocomposites. The data of PLA/PBAT and PLA/PBAT/CNC3 samples prepared through mTSE are adopted from our previous study [43]

\subsection{DSC Analysis}

Fig. 4 and Tab. 2 show DSC thermograms of neat PLA/PBAT blends and PLA/PBAT/CNC blend nanocomposites with $3 \mathrm{wt} \%$ of $\mathrm{CNC}$ prepared with three different mixing strategies and melt mixing methods through mTSE and mIM. The crystallization and melting behaviors of PLA remark its mechanical behavior, heat resistance, and processability [5]. In the cooling cycles, it was observed that the crystallization peak of PLA shifted towards high temperature with the addition of $3 \mathrm{wt} \% \mathrm{CNC}$ in the blends, regardless of mixing strategies and melt mixing methods. This indicated that $\mathrm{CNC}$ acted as nucleation agent for PLA and facilitated the crystallization process. A small shoulder was also seen at the exothermic peaks of blend nanocomposites prepared via the $\mathrm{m} 1$ and $\mathrm{m} 2$ strategies through $\mathrm{mIM}$. This behavior indicated that the CNC may be localized at the PLA or interface, causing the PLA crystallization at high temperature. The blend nanocomposite prepared with $\mathrm{m} 3$ strategy through $\mathrm{mIM}$ did not show a shoulder, but its crystallization peak was narrower than those of $\mathrm{m} 1$ and $\mathrm{m} 2$. Therefore, it can be concluded that PBAT begins to solidify due to CNC localization in the PBAT phase and the crystallization of PLA is accelerated around the solidified PBAT domains. In both methods, when the $\mathrm{m} 2$ strategy is used, the increase in the degree of crystallinity of PLA can be related to the localization of the CNC in the PLA matrix, and this result confirms the rheology and morphology results. In second heating, double melting peak were detected for the m1-mIM and m2-mTSE. This phenomenon is attributed to either the low temperature peak due to the remaining solvent or to the coexistence of two crystal structures: less perfect crystals with smaller lamella thickness, and to reorganize into crystals with 
higher structural perfection, before re-melting at higher temperature [47]. Moreover, in the second heating cycles, a cold crystallization behavior was not observed in the blend, regardless of mixing strategies and melt mixing method. This may be due to the effect of $\mathrm{CNC}$ addition on the enhanced crystallization of PLA during the cooling cycle.
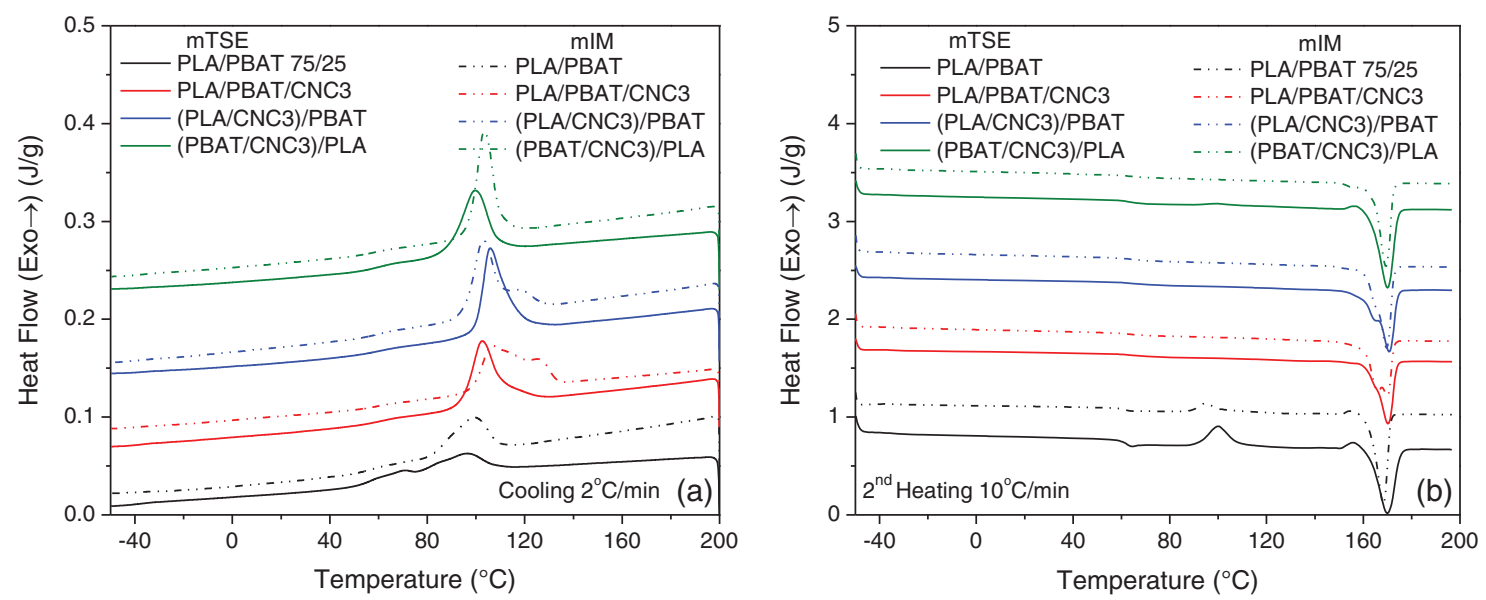

Figure 4: (a) Cooling and (b) second heating scans of the blends and blend nanocomposites prepared with different processing and three mixing strategies. The data of PLA/PBAT and PLA-PBAT-CNC3-m1 samples prepared through mTSE are adopted from our previous study [43]

Table 2: DSC data of samples prepared with different processing and three mixing strategies acquired from cooling and second heating scans

\begin{tabular}{|c|c|c|c|c|c|c|c|c|c|c|c|c|c|c|}
\hline & \multicolumn{7}{|c|}{ mTSE } & \multicolumn{7}{|c|}{ mIM } \\
\hline & \multicolumn{3}{|c|}{ Cooling } & \multicolumn{4}{|c|}{ 2nd heating } & \multicolumn{3}{|c|}{ Cooling } & \multicolumn{4}{|c|}{ 2nd heating } \\
\hline & $\mathrm{Tg}$ & $\mathrm{Tc}$ & $\mathrm{X} \%$ & $\mathrm{Tg}$ & Tcc & $\mathrm{Tm}$ & $\mathrm{X} \%$ & $\mathrm{Tg}$ & $\mathrm{Tc}$ & $\mathrm{X} \%$ & $\mathrm{Tg}$ & Tcc & $\mathrm{Tm}$ & $\mathrm{X} \%$ \\
\hline $75 / 25$ & 55 & 96 & 17 & 61 & 100 & 170 & 30 & 56 & 99 & 27 & 62 & 95 & 168 & 46 \\
\hline $\mathrm{m} 1$ & 58 & 103 & 42 & 66 & - & 170 & 42 & 56 & 107 & 46 & 66 & - & 170 & 48 \\
\hline $\mathrm{m} 2$ & 58 & 106 & 46 & 66 & - & 171 & 48 & 56 & 103 & 49 & 65 & - & 170 & 52 \\
\hline $\mathrm{m} 3$ & 59 & 100 & 40 & 63 & - & 170 & 51 & 56 & 103 & 43 & 66 & - & 169 & 46 \\
\hline
\end{tabular}

\section{$3.5 D M A$}

Fig. 5 shows the storage modulus $\left(\mathrm{E}^{\prime}\right)$ and loss factor $(\tan \delta)$ of neat PLA/PBAT blends and PLA/PBAT/ $\mathrm{CNC}$ blend nanocomposites including $3 \mathrm{wt} \%$ of $\mathrm{CNC}$ in the temperature range of $25-130^{\circ} \mathrm{C}$. As seen in Fig. 5a, all E' curves exhibited a plateau and then began to decrease rapidly after $60^{\circ} \mathrm{C}$ due to the glass transition of PLA. It is also clearly observed that the storage modulus E' rises in the temperature range of $80-100^{\circ} \mathrm{C}$ due to the cold crystallization of PLA. Fig. $5 \mathrm{~b}$ indicated that $3 \mathrm{wt} \% \mathrm{CNC}$ addition slightly shifted the $\tan \delta$ peak to a higher temperature and decreased the intensity of $\tan \delta$ peak. This result corresponds to increase in the $T_{\mathrm{g}}$ value of blend nanocomposites due to the increased stiffness of the blend with the $\mathrm{CNC}$ addition. 

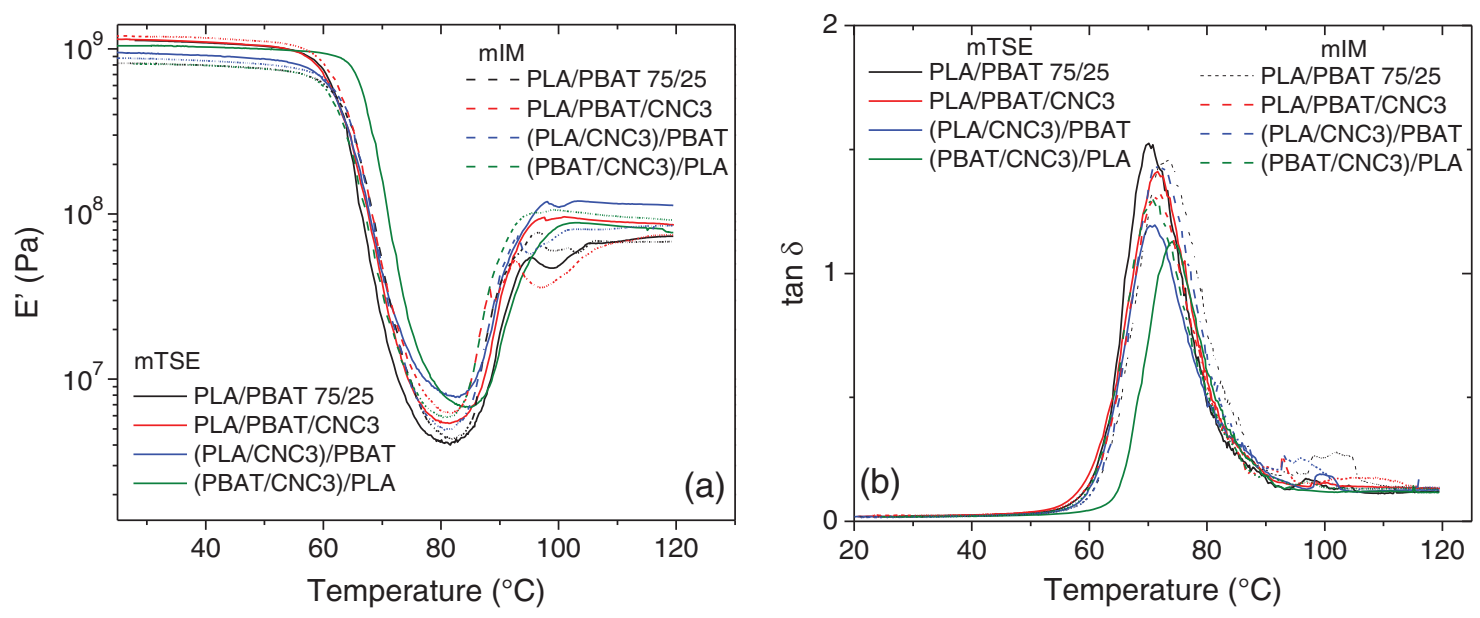

Figure 5: (a) Storage modulus $E^{\prime}$ and (b) Loss factor ( $\tan \delta$ ) curves of the blends and blend nanocomposites prepared via different processing methods and three mixing strategies. The data of PLA/PBAT and PLA/PBAT/CNC3 samples prepared through mTSE are adopted from our previous study [43]

Fig. 6 illustrates the storage moduli of samples at 45 and $85^{\circ} \mathrm{C}$ which, respectively, correspond to the glassy and rubbery regions of PLA. In the glassy region, the blends and blend nanocomposites prepared with both methods and three mixing strategies show a similar storage modulus value. It can be deduced that the $\mathrm{CNC}$ addition did not affect the elastic modulus of samples, significantly at glassy region. On the other hand, $\mathrm{E}^{\prime}$ values of blends increased with the $\mathrm{CNC}$ addition in rubbery region and the influence of $\mathrm{CNC}$ nanoparticles became more decisive.
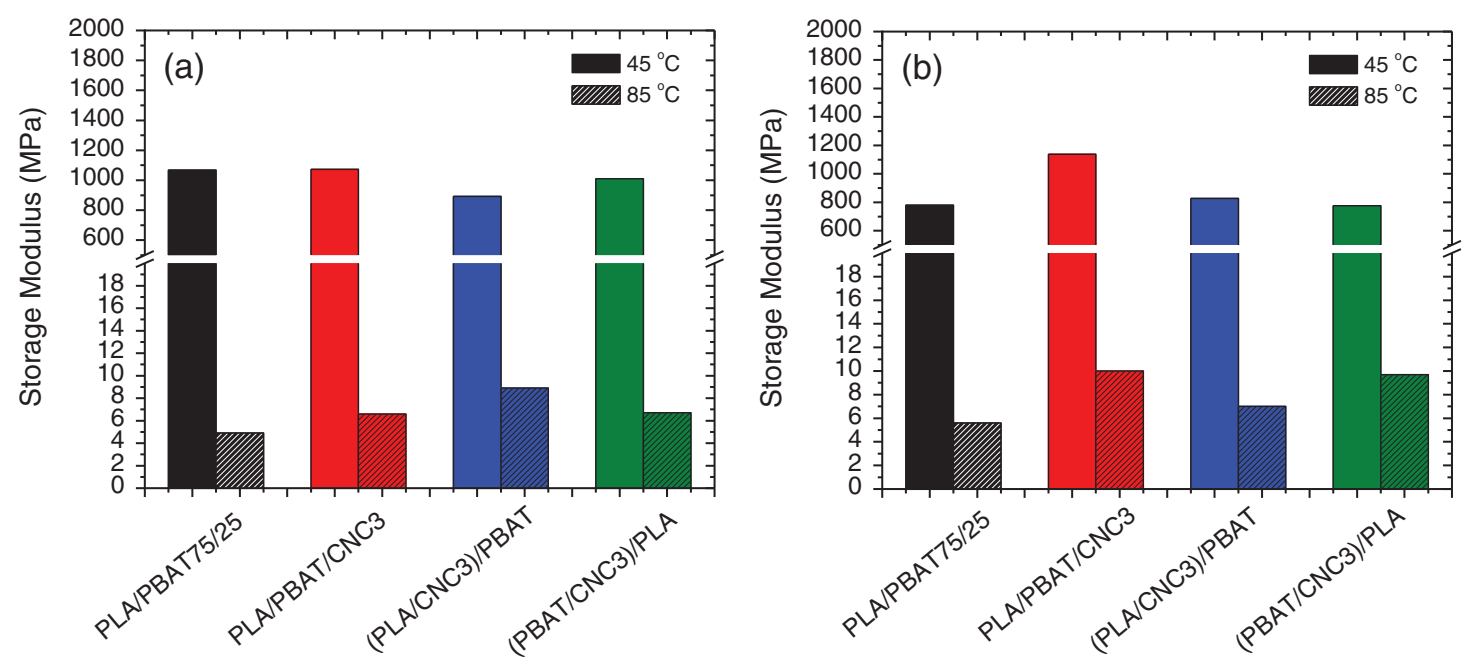

Figure 6: Comparison of the storage modulus $\left(E^{\prime}\right)$ of blends and blend nanocomposites prepared through three mixing strategies using (a) mTSE and (b) mIM. The data of PLA/PBAT and PLA/PBAT/CNC3 samples prepared through mTSE are adopted from our previous study [43]

\subsection{Mechanical Analysis}

Figs. 7 and 8 show the tensile and impact properties of blend and blend nanocomposites prepared by using three different mixing strategies with mTSE and mIM. In the blends prepared with both processing 
techniques, a decrease in tensile strength and modulus was observed compared to neat PLA. It was found that the tensile strength of the blend prepared with mIM was lower than that prepared with MTSE. This may be related to the lower viscosity of the mIM blends due to longer thermal exposure during melt blending as discussed in the previous section. The elongation at break, energy at break and izod impact strength of the PLA/PBAT blend prepared with mTSE increased compared to neat PLA due to the soft structure of PBAT. However, as noted above, a reduction was observed compared to neat PLA. The tensile strength and modulus of blend nanocomposites containing $3 \mathrm{wt} \%$ of CNC prepared with different strategies, were shown to be lower than the neat PLA except for the modulus of the (PBAT/CNC3)/PLA (m3 strategy) prepared with mIM. Apart from the sample prepared with the M3 strategy and mTSE, a decrease in the elongation at break of the blend nanocomposites was observed due to the stiffness of CNC. Izod impact tests revealed that the toughness decreased in blend nanocomposites containing $3 \mathrm{wt} \%$ of CNC prepared with mTSE. On the other hand, the toughness of the blend nanocomposites prepared with the mIM increased compared to the neat blend.
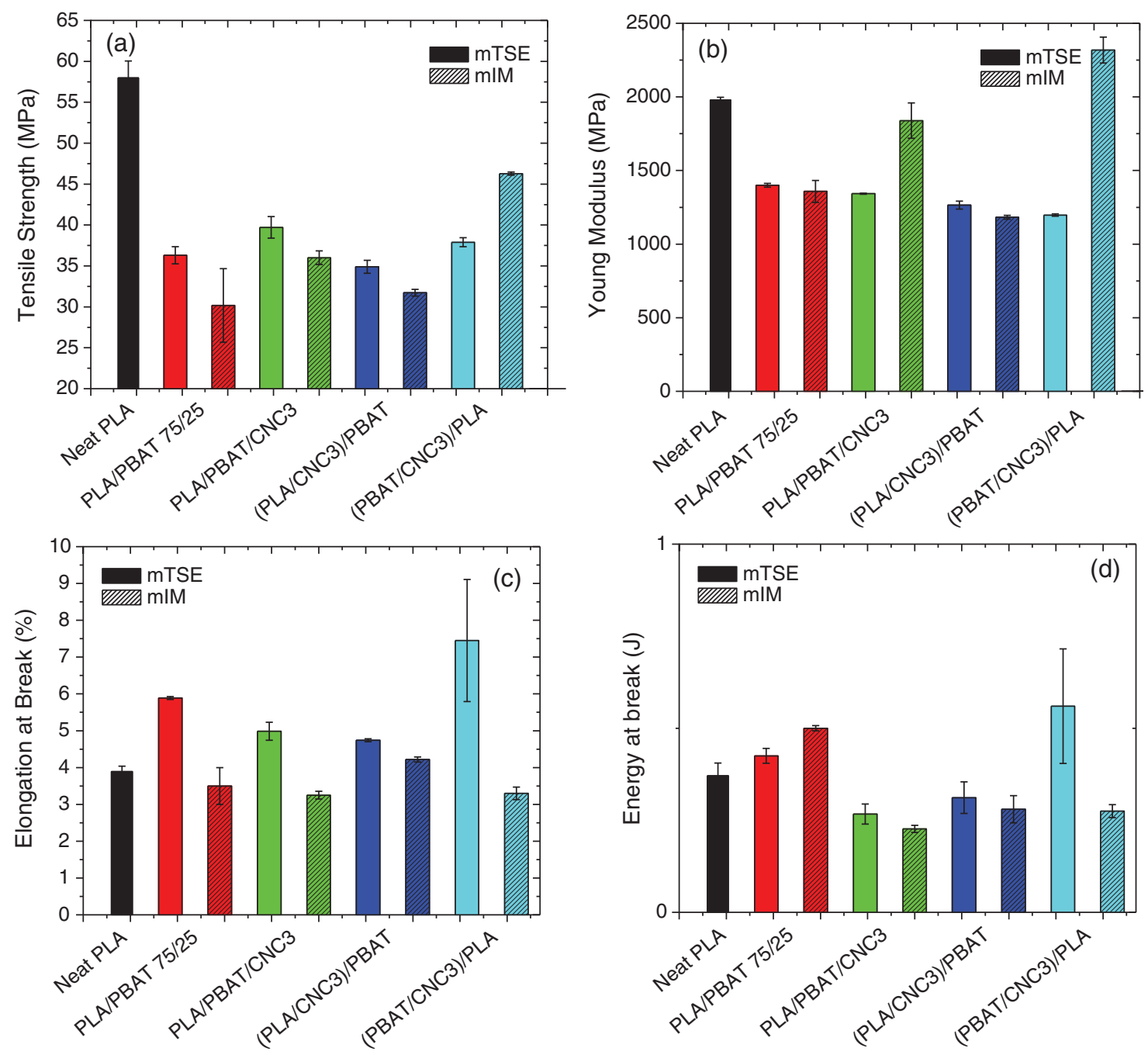

Figure 7: Tensile strength (a), Young modulus (b), elongation at break (c), and energy at break (d) of blends and blend nanocomposites prepared through three mixing strategies using mTSE and mIM. The data of PLA/PBAT and PLA/PBAT/CNC3 samples prepared through mTSE are adopted from our previous study [43] 


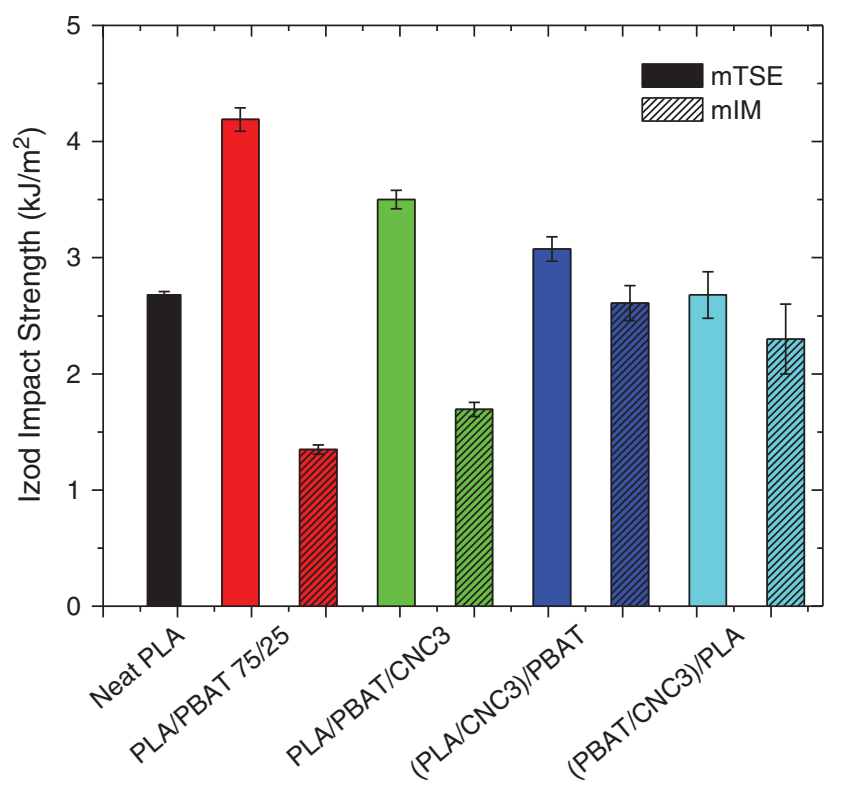

Figure 8: Izod impact strength of blends and blend nanocomposites with different processing and mixing strategies. The data of PLA/PBAT and PLA/PBAT/CNC3 samples prepared through mTSE are adopted from our previous study [43]

It is evident that a small amount of residual solvent could make the samples quite brittle in as much as that compression molding is not possible. Therefore, we specifically emphasize in this study that the expected enhancement in the impact strength, ductility and toughness of PLA-based blends were not attained in the solvent-based sample preparation methods even after a carefully drying the samples, despite significant rheological improvements were obtained. It could be concluded that in order to achieve relevant enhancements in the mechanical properties, solvent-free preparation methods should be utilized. This conclusion obviously refers to the importance of melt processing methods in the manufacturing of thermoplastic nanocomposites despite the great challenge in CNC dispersion. Therefore, promising CNC surface modification could be a solution in improving the CNC dispersion as well as the mechanical properties concurrently. Hence besides the mechanical properties, the barrier properties of the developed system could also significantly be affected $[48,49]$.

\section{Conclusion}

The current study investigated the influence of CNC incorporation on the morphological, rheological, crystallization, and mechanical properties of the PLA/PBAT blend nanocomposites. The main objective was to investigate how different preparation methods affect the dispersion and localization of CNC and hence the properties of PLA/PBAT/CNC blend nanocomposites. The corresponding nanocomposites were prepared through dilution of solution casted masterbatch using a twin-screw extruder or an internal mixer. The effect of mixing strategies on the physical properties of samples was also studied. According to the Young's model, CNC particles thermodynamically preferred to be inside the dispersed PBAT. However, SEM images revealed that the localization of CNCs varied depending on the mixing strategies and processing methods. CNC has been found to be more dispersed in the PLA phase or interface than PBAT. Network of CNC was formed only in M2 strategy and mIM according to the rheological analysis. In the mTSE prepared nanocomposites, however, rheological properties were not significantly enhanced possibly due to the shorter residence time in the extruder than in the internal mixer required to break the CNC agglomerations. Thus, CNC network did not form in the TSE method. Regardless of mixing 
strategies and processing method, CNC showed crystallization accelerating effect for PLA. Although CNC addition affected the morphology, rheology and crystallization properties of PLA/PBAT blends, the mechanical properties of PLA did not improve due to the presence of small amount of solvent in the samples. Consequently, it has been concluded that simultaneously melt mixing methods should be utilized to enhance the mechanical properties significantly by also employing the appropriate surface modification of CNC nanoparticles.

Acknowledgement: The authors would like to sincerely thank Professor Seniha Fatma Guner, our TUBITAK 1001 project consultant, and Professors Metin Hayri Acar and Sinan Sen for providing us their lab space to run some of the experiments.

Funding Statement: The authors would like to acknowledge the financial supports by the Scientific and Technological Research Council of Turkey (TUBITAK) in the Context of 1001 Project with the Project No. of $117 \mathrm{M} 238$.

Conflicts of Interest: The authors declare that they have no conflicts of interest to report regarding the present study.

\section{References}

1. Garlotta, D. (2001). A literature review of poly (lactic acid). Journal of Polymers and the Environment, 9(2), 63-84. DOI 10.1023/A:1020200822435.

2. Yu, L., Dean, K., Li, L. (2006). Polymer blends and composites from renewable resources. Progress in Polymer Science, 31(6), 576-602. DOI 10.1016/j.progpolymsci.2006.03.002.

3. Nofar, M., Sacligil, D., Carreau, P. J., Kamal, M. R., Heuzey, M. C. (2019). Poly (lactic acid) blends: Processing, properties and applications. International Journal of Biological Macromolecules, 125, 307-360. DOI 10.1016/j. ijbiomac.2018.12.002.

4. Tsuji, H. (2005). Poly (lactide) stereocomplexes: Formation, structure, properties, degradation, and applications. Macromolecular Bioscience, 5(7), 569-597. DOI 10.1002/mabi.200500062.

5. Saeidlou, S., Huneault, M. A., Li, H., Park, C. B. (2012). Poly (lactic acid) crystallization. Progress in Polymer Science, 37(12), 1657-1677. DOI 10.1016/j.progpolymsci.2012.07.005.

6. Jiang, L., Wolcott, M. P., Zhang, J. (2006). Study of biodegradable polylactide/poly (butylene adipate-coterephthalate) blends. Biomacromolecules, 7(1), 199-207. DOI 10.1021/bm050581q.

7. Yuan, H., Liu, Z., Ren, J. (2009). Preparation, characterization, and foaming behavior of poly (lactic acid)/poly (butylene adipate-co-butylene terephthalate) blend. Polymer Engineering \& Science, 49(5), 1004-1012. DOI 10.1002/pen.21287.

8. Nuzzo, A., Bilotti, E., Peijs, T., Acierno, D., Filippone, G. (2014). Nanoparticle-induced co-continuity in immiscible polymer blends-A comparative study on bio-based PLA-pA11 blends filled with organoclay, sepiolite, and carbon nanotubes. Polymer, 55(19), 4908-4919. DOI 10.1016/j.polymer.2014.07.036.

9. Dil, E. J., Virgilio, N., Favis, B. D. (2016). The effect of the interfacial assembly of nano-silica in poly (lactic acid)/ poly (butylene adipate-co-terephthalate) blends on morphology, rheology and mechanical properties. European Polymer Journal, 85, 635-646. DOI 10.1016/j.eurpolymj.2016.07.022.

10. Lee, H. S., Fasulo, P. D., Rodgers, W. R., Paul, D. R. (2005). TPO based nanocomposites. Part 1. Morphology and mechanical properties. Polymer, 46(25), 11673-11689. DOI 10.1016/j.polymer.2005.09.068.

11. Nofar, M., Heuzey, M. C., Carreau, P. J., Kamal, M. R. (2020). Nanoparticle interactions and molecular relaxation in PLA/PBAT/Nanoclay blends. Experimental Results, 1, E47. DOI 10.1017/exp.2020.54.

12. Kontopoulou, M., Liu, Y., Austin, J. R., Parent, J. S. (2007). The dynamics of montmorillonite clay dispersion and morphology development in immiscible ethylene-propylene rubber/polypropylene blends. Polymer, 48(15), 4520-4528. DOI 10.1016/j.polymer.2007.05.068. 
13. Hong, J. S., Namkung, H., Ahn, K. H., Lee, S. J., Kim, C. (2006). The role of organically modified layered silicate in the breakup and coalescence of droplets in PBT/PE blends. Polymer, 47(11), 3967-3975. DOI 10.1016/j. polymer.2006.03.077.

14. Fenouillot, F., Cassagnau, P., Majesté, J. C. (2009). Uneven distribution of nanoparticles in immiscible fluids: Morphology development in polymer blends. Polymer, 50(6), 1333-1350. DOI 10.1016/j.polymer.2008.12.029.

15. Taguet, A., Cassagnau, P., Lopez-Cuesta, J. M. (2014). Structuration, selective dispersion and compatibilizing effect of (nano) fillers in polymer blends. Progress in Polymer Science, 39(8), 1526-1563. DOI 10.1016/j. progpolymsci.2014.04.002.

16. Ock, H. G., Kim, D. H., Ahn, K. H., Lee, S. J., Maia, J. M. (2016). Effect of organoclay as a compatibilizer in poly (lactic acid) and natural rubber blends. European Polymer Journal, 76, 216-227. DOI 10.1016/j. eurpolymj.2016.01.042.

17. Gubbels, F., Jérôme, R., Vanlathem, E., Deltour, R., Blacher, S. et al. (1998). Kinetic and thermodynamic control of the selective localization of carbon black at the interface of immiscible polymer blends. Chemistry of Materials, 10(5), 1227-1235. DOI 10.1021/cm970594d.

18. Bai, L., Sharma, R., Cheng, X., Macosko, C. W. (2018). Kinetic control of graphene localization in co-continuous polymer blends via melt compounding. Langmuir, 34(3), 1073-1083. DOI 10.1021/acs.langmuir.7b03085.

19. Taghizadeh, A., Favis, B. D. (2013). Carbon nanotubes in blends of polycaprolactone/thermoplastic starch. Carbohydrate Polymers, 98(1), 189-198. DOI 10.1016/j.carbpol.2013.05.024.

20. Göldel, A., Kasaliwal, G., Pötschke, P. (2009). Selective localization and migration of multiwalled carbon nanotubes in blends of polycarbonate and poly (styrene-acrylonitrile). Macromolecular Rapid Communications, 30(6), 423-429. DOI 10.1002/marc.200800549.

21. Ko, S. W., Hong, M. K., Park, B. J., Gupta, R. K., Choi, H. J. et al. (2009). Morphological and rheological characterization of multi-walled carbon nanotube/PLA/PBAT blend nanocomposites. Polymer Bulletin, 63(1), 125-134. DOI 10.1007/s00289-009-0072-9.

22. Dil, E. J., Arjmand, M., Li, Y., Sundararaj, U., Favis, B. D. (2016). Assembling copper nanowires at the interface and in discrete phases in PLA-based polymer blends. European Polymer Journal, 85, 187-197. DOI 10.1016/j. eurpolymj.2016.09.053.

23. Adrar, S., Habi, A., Ajji, A., Grohens, Y. (2018). Synergistic effects in epoxy functionalized graphene and modified organo-montmorillonite PLA/PBAT blends. Applied Clay Science, 157, 65-75. DOI 10.1016/j.clay.2018.02.028.

24. Habibi, Y., Lucia, L. A., Rojas, O. J. (2010). Cellulose nanocrystals: Chemistry, self-assembly, and applications. Chemical Reviews, 110(6), 3479-3500. DOI 10.1021/cr900339w.

25. Azizi Samir, M. A. S., Alloin, F., Dufresne, A. (2005). Review of recent research into cellulosic whiskers, their properties and their application in nanocomposite field. Biomacromolecules, 6(2), 612-626. DOI 10.1021/ bm0493685.

26. Moon, R. J., Martini, A., Nairn, J., Simonsen, J., Youngblood, J. (2011). Cellulose nanomaterials review: Structure, properties and nanocomposites. Chemical Society Reviews, 40(7), 3941-3994. DOI 10.1039/c0cs00108b.

27. Oksman, K., Aitomäki, Y., Mathew, A. P., Siqueira, G., Zhou, Q. et al. (2016). Review of the recent developments in cellulose nanocomposite processing. Composites Part A: Applied Science and Manufacturing, 83, 2-18. DOI 10.1016/j.compositesa.2015.10.041.

28. Vatansever, E., Arslan, D., Nofar, M. (2019). Polylactide cellulose-based nanocomposites. International Journal of Biological Macromolecules, 137, 912-938. DOI 10.1016/j.ijbiomac.2019.06.205.

29. Khoshkava, V., Kamal, M. R. (2013). Effect of surface energy on dispersion and mechanical properties of polymer/ nanocrystalline cellulose nanocomposites. Biomacromolecules, 14(9), 3155-3163. DOI 10.1021/bm400784j.

30. Arias, A., Heuzey, M. C., Huneault, M. A., Ausias, G., Bendahou, A. (2015). Enhanced dispersion of cellulose nanocrystals in melt-processed polylactide-based nanocomposites. Cellulose, 22(1), 483-498. DOI 10.1007/ s10570-014-0476-z.

31. Bondeson, D., Oksman, K. (2007). Dispersion and characteristics of surfactant modified cellulose whiskers nanocomposites. Composite Interfaces, 14(7-9), 617-630. DOI 10.1163/156855407782106519. 
32. Bagheriasl, D., Safdari, F., Carreau, P. J., Dubois, C., Riedl, B. (2019). Development of cellulose nanocrystalreinforced polylactide: A comparative study on different preparation methods. Polymer Composites, 40(S1), E342-E349. DOI 10.1002/pc.24676.

33. Bagheriasl, D., Carreau, P. J., Riedl, B., Dubois, C. (2018). Enhanced properties of polylactide by incorporating cellulose nanocrystals. Polymer Composites, 39(8), 2685-2694. DOI 10.1002/pc.24259.

34. Bagheriasl, D., Carreau, P. J., Riedl, B., Dubois, C., Hamad, W. Y. (2016). Shear rheology of polylactide (PLA)cellulose nanocrystal (CNC) nanocomposites. Cellulose, 23(3), 1885-1897. DOI 10.1007/s10570-016-0914-1.

35. Arslan, D., Vatansever, E., Sarul, D. S., Kahraman, Y., Gunes, G. et al. (2020). Effect of preparation method on the properties of polylactide/cellulose nanocrystal nanocomposites. Polymer Composites, 41(10), 4170-4180. DOI $10.1002 / \mathrm{pc} .25701$.

36. Mohammadi, M., Bruel, C., Heuzey, M. C., Carreau, P. J. (2020). CNC dispersion in PLA and PBAT using two solvents: Morphological and rheological properties. Cellulose, 27(17), 9877-9892. DOI 10.1007/s10570-020-03460-8.

37. Mohammadi, M., Heuzey, M. C., Carreau, P. J., Taguet, A. (2021). Morphological and rheological properties of PLA. PBAT, and PLA/PBAT Blend Nanocomposites Containing CNCs. Nanomaterials, 11(4), 857. DOI 10.3390/nano11040857.

38. Pracella, M., Haque, M. M. U., Puglia, D. (2014). Morphology and properties tuning of PLA/cellulose nanocrystals bio-nanocomposites by means of reactive functionalization and blending with PVAc. Polymer, 55(16), 3720-3728. DOI 10.1016/j.polymer.2014.06.071.

39. Shakouri, Z., Nazockdast, H. (2018). Microstructural development and mechanical performance of PLA/TPU blends containing geometrically different cellulose nanocrystals. Cellulose, 25(12), 7167-7188. DOI 10.1007/ s10570-018-2061-3.

40. Bitinis, N., Verdejo, R., Bras, J., Fortunati, E., Kenny, J. M. et al. (2013). Poly (lactic acid)/natural rubber/cellulose nanocrystal bionanocomposites part I. processing and morphology. Carbohydrate Polymers, 96(2), 611-620. DOI 10.1016/j.carbpol.2013.02.068.

41. Heshmati, V., Kamal, M. R., Favis, B. D. (2018). Tuning the localization of finely dispersed cellulose nanocrystal in poly (lactic acid)/bio-polyamide11 blends. Journal of Polymer Science Part B: Polymer Physics, 56(7), 576587. DOI 10.1002/polb.24563.

42. Heshmati, V., Kamal, M. R., Favis, B. D. (2018). Cellulose nanocrystal in poly (lactic acid)/polyamide11 blends: Preparation, morphology and co-continuity. European Polymer Journal, 98, 11-20. DOI 10.1016/j. eurpolymj.2017.10.027.

43. Sarul, D. S., Arslan, D., Vatansever, E., Kahraman, Y., Durmus, A. et al. (2021). Preparation and characterization of PLA/PBAT/CNC blend nanocomposites. Colloid and Polymer Science, 299, 987-998. DOI 10.1007/s00396021-04822-9.

44. Beuguel, Q., Tavares, J. R., Carreau, P. J., Heuzey, M. C. (2018). Ultrasonication of spray-and freeze-dried cellulose nanocrystals in water. Journal of Colloid and Interface Science, 516, 23-33. DOI 10.1016/j. jcis.2018.01.035.

45. Yasuniwa, M., Tsubakihara, S., Iura, K., Ono, Y., Dan, Y. et al. (2006). Crystallization behavior of poly (L-lactic acid). Polymer, 47(21), 7554-7563. DOI 10.1016/j.polymer.2006.08.054.

46. Abbasi, S., Carreau, P. J., Derdouri, A. (2010). Flow induced orientation of multiwalled carbon nanotubes in polycarbonate nanocomposites: Rheology, conductivity and mechanical properties. Polymer, 51(4), 922-935. DOI 10.1016/j.polymer.2009.12.041.

47. Kumar, M., Mohanty, S., Nayak, S. K., Parvaiz, M. R. (2010). Effect of glycidyl methacrylate (GMA) on the thermal, mechanical and morphological property of biodegradable PLA/PBAT blend and its nanocomposites. Bioresource Technology, 101(21), 8406-8415. DOI 10.1016/j.biortech.2010.05.075.

48. Li, F., Zhang, C., Weng, Y. (2020). Improvement of the Gas barrier properties of PLA/OMMT films by regulating the interlayer spacing of OMMT and the crystallinity of PLA. ACS Omega, 5(30), 18675-18684. DOI 10.1021/ acsomega.0c01405.

49. Wang, N., Zhang, C., Weng, Y. (2021). Enhancing gas barrier performance of polylactic acid/lignin composite films through cooperative effect of compatibilization and nucleation. Journal of Applied Polymer Science, 138(15), 50199. DOI 10.1002/app.50199. 